\title{
THE COEFFICIENTS OF CERTAIN INFINITE PRODUCTS
}

\author{
MORRIS NEWMAN
}

Introduction. The identities

$$
\begin{aligned}
& \prod_{n=1}^{\infty}\left(1-x^{n}\right)=\sum_{n=-\infty}^{\infty}(-1)^{n} x^{\left(3 n^{2}+n\right) / 2} \\
& \prod_{n=1}^{\infty}\left(1-x^{n}\right)^{3}=\sum_{n=0}^{\infty}(-1)^{n}(2 n+1) x^{\left(n^{2}+n\right) / 2}
\end{aligned}
$$

are classical, (a) being the so-called "pentagonal number theorem" of Euler, and (b) being an identity of Jacobi's, well known in the theory of elliptic modular functions. It is remarkable that for no other values of $r$ are the coefficients of $\prod_{n=1}^{\infty}\left(1-x^{n}\right)^{r}$ known explicitly. It will be the purpose of this paper to give various recursion formulas and identities for these coefficients, which are consequences of identities between functions on certain modular subgroups. In particular, the coefficients will be investigated thoroughly for $r=2,4,6$. A typical theorem is that every integer occurs as the modulus of the coefficients for $r=2$.

1. Preliminary identities. In what follows all sums will be extended from 0 to $\infty$ and all products from 1 to $\infty$, unless otherwise indicated. We denote the coefficient of $x^{n}$ in $\Pi\left(1-x^{n}\right)^{r}$ by $p_{r}(n)$; i.e.,

$$
\Pi\left(1-x^{n}\right)^{r}=\sum p_{r}(n) x^{n} .
$$

Noting that $\Pi\left(1-x^{n}\right)^{r}=\Pi\left(1-x^{n}\right)^{a} \cdot \Pi\left(1-x^{n}\right)^{r-a}$, we obtain the identity

$$
p_{r}(n)=\sum_{j=0}^{n} p_{a}(j) p_{r-a}(n-j) .
$$

Differentiating (1.1) logarithmically, we also obtain

$$
n p_{r}(n)=-r \sum_{j=1}^{n} \sigma(j) p_{r}(n-j), \quad \sigma(j)=\sum_{d \mid j} d .
$$

(1.3) is especially suited to numerical calculation.

Using (a), (b), and (1.2), we can obtain the following explicit formulas for $p_{2}(n), p_{4}(n), p_{6}(n)$ :

$$
\text { (1.4) } \quad p_{2}(n)=\sum_{\left(3 x^{2} \pm x\right) / 2+\left(3 y^{2} \pm y\right) / 2=n}(-1)^{x+y},
$$

Received by the editors October 15, 1952. 


$$
\begin{aligned}
& p_{4}(n)=\sum_{\left(x^{2}+x\right) / 2+\left(3 y^{2} \pm y\right) / 2=n}(-1)^{x+y}(2 x+1), \\
& p_{6}(n)=\sum_{\left(x^{2}+x\right) / 2+\left(y^{2}+y\right) / 2=n}(-1)^{x+y}(2 x+1)(2 y+1) .
\end{aligned}
$$

It is understood in formulas (1.4)-(1.6) that $(x, y)$ runs over all non-negative integral solutions of the defining equations.

These formulas, though complicated, are arithmetically enlightening and will be studied in detail. We set $\langle x\rangle=[x+1 / 2]$, so that $\langle x\rangle$ is the integer nearest to $x$.

A little consideration shows that we may rewrite formulas (1.4)(1.6) as follows:

$$
\begin{aligned}
& p_{2}(n)=\sum_{u^{2}+v^{2}=24 n+2}(-1)^{\langle(u+v) / 6\rangle}, \\
& p_{4}(n)=\sum_{u^{2}+3 v^{2}=24 n+4, u, v \text { odd }}(-1)^{[(u+3 v) / 6]} v, \\
& p_{6}(n)=-\sum_{u^{2}+v^{2}=8 n+2}(-1)^{(u+v) / 2} u v .
\end{aligned}
$$

It is understood in formulas $(1.7)-(1.9)$ that $(u, v)$ runs over all positive integral solutions of the defining equations.

Let $a=\prod_{p \mid a} p^{e_{p}}$ be the canonical decomposition of $a$. If $e_{p}$ is odd for some $p \equiv-1$ (4), then the equation $x^{2}+y^{2}=a$ has no solutions in integers. If $e_{p}$ is odd for some $p \equiv-1$ (3), then the equation $x^{2}+3 y^{2}=a$ has no solutions in integers. We can say therefore:

(1.10) Let $r=2,4,6$. Let $24 n / r+1=\prod_{p \mid 24 n / r+1} p^{\alpha_{p}}$ be the canonical decomposition of $24 n / r+1$. If $e_{p}$ is odd for some $p \equiv-1\left(m_{r}\right)$, where $m_{r}=4,3,4$ for $r=2,4,6$, then $p_{r}(n)=0$.

Hence we need only consider $n$ such that $24 n / r+1=q_{r} Q_{r}^{2}$, where $q_{r}$ is a product of primes $\equiv 1\left(m_{r}\right)$ and $Q_{r}$ is a product of primes $\equiv-1\left(m_{r}\right)$.

We note that if $p \equiv-1(4)$, then $x^{2}+y^{2} \equiv 0(p)$ implies $x \equiv y \equiv 0(p)$, and if $p \equiv-1(3)$, then $x^{2}+3 y^{2} \equiv 0(p)$ implies that $x \equiv y \equiv 0(p)$.

Making use of the above, we obtain the following reduction formulas:

$$
\begin{aligned}
& p_{2}\left[\left(q_{2} Q_{2}^{2}-1\right) / 12\right]=\sum_{u^{2}+v^{2}=2 q_{2}}(-1)^{\left\langle Q_{2}(u+v) / \sigma\right\rangle}, \\
& p_{4}\left[\left(q_{4} Q_{4}^{2}-1\right) / 6\right]=Q_{4} \sum_{u^{2}+3 v^{2}=4 q_{4}, u, v \text { odd }}(-1)^{\left[Q_{4}(u+3 v) / 6\right]} . \\
& p_{6}\left[\left(q_{6} Q_{6}^{2}-\right) / 4\right]=Q_{6}^{2} p_{6}\left[\left(q_{6}-1\right) / 4\right] .
\end{aligned}
$$

In particular, 


$$
\begin{aligned}
p_{2}\left[\left(Q_{2}^{2}-1\right) / 12\right] & =(-1)^{\left\langle Q_{2} / 3\right\rangle}, \\
p_{4}\left[\left(Q_{4}^{2}-1\right) / 6\right] & =(-1)^{\left[2 Q_{4} / 3\right]} Q_{4}, \\
p_{6}\left[\left(Q_{6}^{2}-1\right) / 4\right] & =Q_{6}^{2} .
\end{aligned}
$$

A similar set of explicit formulas can be written down when $q_{r}$ is a prime. We note only that if $q_{2}$ is a prime, then

$$
p_{2}\left[\left(q_{2} Q_{2}^{2}-1\right) / 12\right]=2(-1)\left\langle Q_{2}(A+B) / 6\right\rangle,
$$

where $A, B$ are the uniquely determined positive integers such that $2 q_{2}=A^{2}+B^{2}$.

2. The principal identities. We are going to extend the scope of the previous identities by recursion formulas derived from identities between modular functions. The following theorem has been proved by the author in his paper [1]:1

THEOREM 1. Suppose that $r$ is even, $0<r \leqq 24$. Let $p$ be a prime such that $r(p-1) \equiv 0(24)$. Set $\delta=r(p-1) / 24$. Then

$$
\sum p_{r}(n p+\delta) x^{n}=p_{r}(\delta) \Pi\left(1-x^{n}\right)^{r}-p^{r / 2-1} x^{\delta} \Pi\left(1-x^{n p}\right)^{r}
$$

By comparing coefficients of corresponding powers of $x$, we find

$$
p_{r}(n p+\delta)=p_{r}(\delta) p_{r}(n)-p^{r / 2-1} p_{r}((n-\delta) / p),{ }^{2}
$$

with the convention that $p_{r}(x)$ is zero if $x$ is not an integer.

We propose to derive some more identities of this type for $r=2,4,6$ and for various associated p's. For this purpose we shall require a number-theoretic lemma, the proof of which is straightforward and will be omitted.

Lemma 1. Suppose that $r$ is 2, 4, or 6. Let $p$ be a prime such that $r(p+1) \equiv 0 \quad(24) . \quad$ Set $\Delta=r\left(p^{2}-1\right) / 24, \quad \lambda_{n}=\Delta-p[\Delta / p]+n p, \quad n$ $=0,1, \cdots$. Then $[\Delta / p]$ is the least value of $n$ for which $\lambda_{n}$ is the sum of two pentagons $(r=2)$; the sum of a triangle and a pentagon $(r=4)$; the sum of two triangles $(r=6)$.

Lemma 1 leads easily to a proof that the functions $S_{r}$ defined in [1] are constant, under the conditions imposed above. ${ }^{3}$ We are thus led to the following theorem:

\section{TheOREM 2. With the notation of Lemma 1, we have}

${ }^{1}$ Numbers in brackets refer to the bibliography at the end of the paper.

2 The case $r=24$ reduces to Mordell's identity for Ramanujan's function $\tau(n)$.

${ }^{3}$ The coefficients of the pole terms are essentially $\operatorname{p}_{r}\left(\lambda_{n}\right), n=0,1, \cdots,[\Delta / p]-1$. These coefficients are of necessity zero, in view of the formulas (1.4)-(1.6). 


$$
\sum p_{r}(n p+\Delta) x^{n}=(-p)^{r / 2-1} \prod\left(1-x^{n p}\right)^{r} .
$$

By comparing coefficients of corresponding powers of $x$, we find

$$
p_{r}(n p+\Delta)=(-p)^{r / 2-1} p_{r}(n / p) .
$$

3. Deductions from the principal identities. We shall consider recursion formulas (2.2) and (2.4) more closely. It will be understood that a formula derived from a theorem is subject to the hypotheses of that theorem.

We see from (2.4) that

$$
\operatorname{pr}(n p+\Delta)=0, \quad \text { if }(n, p)=1 .
$$

Setting $n=p g_{t}, \quad g_{t+1}=p^{2} g_{t}+\Delta \quad(t=0,1, \cdots)$, and $A_{t}=p_{r}\left(g_{t}\right)$, formula (2.4) reads $A_{t+1}=(-p)^{r / 2-1} A_{t}$. This implies that $p_{r}\left(g_{0} p^{2 t}\right.$ $\left.+r\left(p^{2 t}-1\right) / 24\right)=(-p)^{(r / 2-1)} t p_{r}\left(g_{0}\right)$. In particular, choosing $g_{0}=0,1,2$ we obtain

$$
\begin{aligned}
p_{r}\left(r\left(p^{2 t}-1\right) / 24\right) & =(-p)^{(r / 2-1) t} \\
p_{r}\left(p^{2 t}+r\left(p^{2 t}-1\right) / 24\right) & =-r(-p)^{(r / 2-1) t} \\
p_{r}\left(2 p^{2 t}+r\left(p^{2 t}-1\right) / 24\right) & =r(r-3) / 2 \cdot(-p)^{(r / 2-1) t} .
\end{aligned}
$$

The recursion formula (2.2) requires a slightly more elaborate discussion. Setting $a=p_{r}(\delta), b=p^{r / 2-1}, n=g_{t}, g_{t+1}=p g_{t}+\delta(t=0,1, \cdots)$, and $A_{t}=p_{r}\left(g_{t}\right),(2.2)$ reads $A_{t+2}-a A_{t+1}+b A_{t}=0$. The solution of this difference equation depends on the nature of the roots of the characteristic equation $x^{2}-a x+b=0$. This in turn depends on the discriminant $a^{2}-4 b$. We are interested in particular in determining when this discriminant vanishes. (1.17) shows that this occurs for $r=2$, and it is easy to verify that it does not occur for $r=4,6$. For $r=2$, then, we obtain

$$
p_{2}\left(g_{0} p^{t}+\left(p^{t}-1\right) / 12\right)=(t+1)\left(p_{2}((p-1) / 12) / 2\right)^{t} \cdot p_{2}\left(g_{0}\right) .
$$

Applying(1.17), we have

$$
p_{2}\left(\left(p^{t}-1\right) / 12\right)=( \pm 1)^{t}(t+1) .
$$

It is clear that (3.6) implies that any integer occurs as the modulus of the coefficients in $\Pi\left(1-x^{n}\right)^{2}$, and does so infinitely often.

For $r=4$, 6 we obtain (with $g_{0}=0$ )

$$
\begin{aligned}
p_{r}\left(r\left(p^{t}-1\right) / 24\right) & =\frac{x_{1}^{t+1}-x_{2}^{t+1}}{x_{1}-x_{2}}, \quad x_{1}+x_{2}=p_{r}(\delta), \\
x_{1} x_{2} & =p^{r / 2-1} .
\end{aligned}
$$


A table of $p_{r}(n), r=2,4,6$, is appended for $1 \leqq n \leqq 25$.

$\begin{array}{rrrrcrrr}n & p_{2}(n) & p_{6}(n) & p_{6}(n) & n & p_{2}(n) & p_{6}(n) & p_{6}(n) \\ 1 & -2 & -4 & -6 & 16 & 2 & 14 & -60 \\ 2 & -1 & 2 & 9 & 17 & 0 & 20 & 0 \\ 3 & 2 & 8 & 10 & 18 & 0 & 2 & -110 \\ 4 & 1 & -5 & -30 & 19 & -2 & 0 & 0 \\ 5 & 2 & -4 & 0 & 20 & -2 & -11 & 81 \\ 6 & -2 & -10 & 11 & 21 & 0 & 20 & 180 \\ 7 & 0 & 8 & 42 & 22 & 0 & -32 & -78 \\ 8 & -2 & 9 & 0 & 23 & -2 & -16 & 0 \\ 9 & -2 & 0 & -70 & 24 & -1 & 0 & 130 \\ 10 & 1 & 14 & 18 & 25 & 0 & -4 & -198 \\ 11 & 0 & -16 & -54 & & & & \\ 12 & 0 & -10 & 49 & & & & \\ 13 & 2 & -4 & 90 & & & & \\ 14 & 3 & 0 & 0 & & & \end{array}$

1. M. Newman, Remarks on some modular identities, Trans. Amer. Math. Soc. vol. 73 (1952) pp. 313-320.

2. K. G. Ramanathan, Identities and congruences of the Ramanujan type, Canadian Journal of Mathematics vol. 2 (1950) pp. 168-178.

National Bureau of Standards 